\title{
A STRUCTURE THEORY OF $(-1,-1)$-FREUDENTHAL KANTOR TRIPLE SYSTEMS
}

\author{
NORIAKI KAMIYA, DANIEL MONDOC ${ }^{\bowtie}$ and SUSUMU OKUBO
}

\author{
(Received 8 April 2009)
}

\begin{abstract}
In this paper we discuss the simplicity criteria of $(-1,-1)$-Freudenthal Kantor triple systems and give examples of such triple systems, from which we can construct some Lie superalgebras. We also show that we can associate a Jordan triple system to any $(\varepsilon, \delta)$-Freudenthal Kantor triple system. Further, we introduce the notion of $\delta$-structurable algebras and connect them to $(-1, \delta)$-Freudenthal Kantor triple systems and the corresponding Lie (super)algebra construction.
\end{abstract}

2000 Mathematics subject classification: primary 17A40, 17B60.

Keywords and phrases: Lie superalgebras, triple systems.

\section{Introduction}

The history of nonassociative algebras seems to have its roots at the end of the 19th century. Hamilton, Cayley, and Hurwitz were the first investigators in this field. They characterized an algebraic image of a generalization of the complex numbers by the concept of quaternion, octonion numbers, and composition algebras. Later generations, for example Artin and Zorn, studied alternative and nearly associative algebras. And of course, as a later generalization, we have the investigation of Jordan and Lie algebras with applications to physics.

Nonassociative algebras are rich in algebraic structures, and they provide important common ground for various branches of mathematics, not only pure algebra and differential geometry, but also representation theory and algebraic geometry. Specially, the concept of nonassociative algebras such as Jordan and Lie (super)algebras plays an important role in many mathematical and physical subjects $[5,10-13,15,27,29$, $43,44,48,51,52]$. We also note that the construction and characterization of these algebras can be expressed in terms of the notion of triple systems $[19,35,46]$ by using

The first author's research was partially supported by Grant-in-Aid for Scientific Research (No. 19540042 (C),(2)), Japan Society for the Promotion of Science. The second author's research was partially supported by Japan Society for the Promotion of Science (No. PE 07601). The third author's research was supported by U.S. Department of Energy Grant No. DE-FG02-91ER40685.

(C) 2009 Australian Mathematical Publishing Association Inc. 0004-9727/2009 \$16.00 
the standard embedding method [23, 37, 38, 47, 50]. In particular, the generalized Jordan triple system of second order (that is, the $(-1,1)$-Freudenthal Kantor triple system) is a useful concept for the construction of simple Lie algebras [13-20, 3134, 36, 49] and Lie superalgebras [6, 23, 26, 28], while the $\delta$-Jordan Lie triple systems play a similar role in the construction of Jordan superalgebras [24, 25, 46]. Two of the present authors have constructed a model of Lie superalgebras $D(2,1 ; \alpha), G(3)$ and $F(4)$ [26].

The purpose of this paper is the main structure theory of this project on applications of our triple systems. We show that we can associate a Jordan triple system to any $(\varepsilon, \delta)$-Freudenthal Kantor triple system and we deal with a property of the associated Jordan triple systems. Further, we introduce the notion of $\delta$-structurable algebras and connect them to $(-1, \delta)$-Freudenthal Kantor triple systems and the corresponding Lie (super)algebra construction.

\section{Definitions and the Jordan triple systems associated with Freudenthal Kantor triple systems}

2.1. $(\epsilon, \delta)$-Freudenthal Kantor triple systems, $\delta$-Lie triple systems and Lie (super)algebras. We are concerned in this paper with triple systems which have finite dimension over a field $\Phi$ of characteristic not equal to 2 or 3 , unless otherwise specified.

In order to make this paper as self-contained as possible, we recall first the definition of a generalized Jordan triple system (GJTS) of second order.

A vector space $V$ over a field $\Phi$ endowed with a trilinear operation $V \times V \times V \rightarrow$ $V,(x, y, z) \longmapsto(x y z)$ is said to be a GJTS of second order if the following conditions are fulfilled:

$$
\begin{gathered}
(a b(x y z))=((a b x) y z)-(x(b a y) z)+(x y(a b z)), \\
K(K(a, b) x, y)-L(y, x) K(a, b)-K(a, b) L(x, y)=0,
\end{gathered}
$$

where $L(a, b) c:=(a b c)$ and $K(a, b) c:=(a c b)-(b c a)$.

A Jordan triple system (JTS) satisfies (2.1) and the condition

$$
(a b c)=(c b a) .
$$

We can generalize the concept of the GJTS of second order as follows (see [13, 14, $17,23,50]$ and the earlier references therein). For $\varepsilon= \pm 1$ and $\delta= \pm 1$, a triple product that satisfies the identities

$$
\begin{gathered}
(a b(x y z))=((a b x) y z)+\varepsilon(x(b a y) z)+(x y(a b z)), \\
K(K(a, b) x, y)-L(y, x) K(a, b)+\varepsilon K(a, b) L(x, y)=0,
\end{gathered}
$$

where

$$
L(a, b) c:=(a b c), \quad K(a, b) c:=(a c b)-\delta(b c a),
$$

is called an $(\varepsilon, \delta)$-Freudenthal Kantor triple system $((\varepsilon, \delta)$-FKTS). 
REMARK 2.1. We note that

$$
K(b, a)=-\delta K(a, b) .
$$

From now on we will mainly deal with this type of triple system. Furthermore, an $(\varepsilon, \delta)$-FKTS is said to be balanced if it satisfies $\operatorname{dim}_{\Phi}\{K(a, b)\}_{\text {span }}=1$.

Triple products are generally denoted $(x y z),\{x y z\},[x y z]$ and $\langle x y z\rangle$ according to context.

REMARK 2.2. We note that the concept of GJTS of second order coincides with that of $(-1,1)$-FKTS. Thus we can construct the simple Lie algebras by means of the standard embedding method [6, 13-17, 23, 26, 28, 33, 50].

REMARK 2.3. We note that the pairs of identities (2.8) and (2.9) are equivalent:

(i) $(a b(x y z))=((a b x) y z)+\varepsilon(x(b a y) z)+(x y(a b z))$,

(ii) $K(K(a, b) x, y)-L(y, x) K(a, b)+\varepsilon K(a, b) L(x, y)=0$;

and

$$
\begin{gathered}
\text { (i) } \quad[L(a, b), L(x, y)]=L((a b x), y)+\varepsilon L(x,(b a y)), \\
\text { (iii) } \quad K(K(a, b) x, y)-K((y x a), b)-K(a,(y x b))=0,
\end{gathered}
$$

where $\varepsilon= \pm 1, \delta= \pm 1$ and $L(a, b), K(a, b)$ are defined by (2.6). Indeed, from (i) and (2.8) it follows that (2.9) holds. Conversely, from (i) and (2.9) it follows that (2.8) holds.

REMARK 2.4. For an $(\varepsilon, \delta)$-FKTS $U$ we denote

$$
S(a, b):=L(a, b)+\varepsilon L(b, a), \quad A(a, b):=L(a, b)-\varepsilon L(b, a),
$$

where $L(a, b)$ is defined by (2.6).

REMARK 2.5. We note that

$$
S(a, b)=\varepsilon S(b, a)
$$

Then $S(a, b)(A(a, b))$ is a derivation (anti-derivation) of $U$.

Indeed, we note that the following identities are valid:

$$
\begin{aligned}
{[S(a, b), L(c, d)] } & =L(S(a, b) c, d)+L(c, S(a, b) d), \\
{[A(a, b), L(c, d)] } & =L(A(a, b) c, d)-L(c, A(a, b) d) .
\end{aligned}
$$

For $\delta= \pm 1$, a triple system $(a, b, c) \mapsto[a b c], a, b, c \in V$ is called a $\delta$-Lie triple system $(\delta$-LTS) if the following three identities are satisfied:

$$
\begin{gathered}
{[a b c]=-\delta[b a c],} \\
{[a b c]+[b c a]+[c a b]=0,} \\
{[a b[x y z]]=[[a b x] y z]+[x[a b y] z]+[x y[a b z]],}
\end{gathered}
$$

where $a, b, x, y, z \in V$. A 1-LTS is an LTS while a (-1)-LTS is an anti-LTS, by [14]. 
Proposition $2.6[14,23]$. Let $U(\varepsilon, \delta)$ be an $(\varepsilon, \delta)$-FKTS. If $J$ is an endomorphism of $U(\varepsilon, \delta)$ such that $J\langle x y z\rangle=\langle J x J y J z\rangle$ and $J^{2}=-\varepsilon \delta \mathrm{Id}$, then $(U(\varepsilon, \delta),[x y z])$ is an LTS (if $\delta=1$ ) or an anti-LTS (if $\delta=-1$ ) with respect to the product

$$
[x y z]:=\langle x J y z\rangle-\delta\langle y J x z\rangle+\delta\langle x J z y\rangle-\langle y J z x\rangle .
$$

Corollary 2.7. Let $U(\varepsilon, \delta)$ be an $(\varepsilon, \delta)$-FKTS. Then the vector space $T(\varepsilon, \delta)=$ $U(\varepsilon, \delta) \oplus U(\varepsilon, \delta)$ becomes an LTS (if $\delta=1$ ) or an anti-LTS (if $\delta=-1$ ) with respect to the triple product defined by

$$
\left[\left(\begin{array}{l}
a \\
b
\end{array}\right)\left(\begin{array}{l}
c \\
d
\end{array}\right)\left(\begin{array}{l}
e \\
f
\end{array}\right)\right]=\left(\begin{array}{cc}
L(a, d)-\delta L(c, b) & \delta K(a, c) \\
-\varepsilon K(b, d) & \varepsilon(L(d, a)-\delta L(b, c))
\end{array}\right)\left(\begin{array}{l}
e \\
f
\end{array}\right) .
$$

Thus we can obtain the standard embedding Lie algebra (if $\delta=1$ ) or Lie superalgebra (if $\delta=-1$ ), $L(\varepsilon, \delta)=D(T(\varepsilon, \delta), T(\varepsilon, \delta)) \oplus T(\varepsilon, \delta)$, associated to $T(\varepsilon, \delta)$ where $D(T(\varepsilon, \delta), T(\varepsilon, \delta))$ is the set of inner derivations of $T(\varepsilon, \delta)$, that is,

$$
\begin{gathered}
D(T(\varepsilon, \delta), T(\varepsilon, \delta)):=\left\{\left(\begin{array}{cc}
L(a, b) & \delta K(c, d) \\
-\varepsilon K(e, f) & \varepsilon L(b, a)
\end{array}\right)\right\}_{\mathrm{span}}, \\
T(\varepsilon, \delta):=\left\{\left(\begin{array}{l}
x \\
y
\end{array}\right) \mid x, y \in U(\varepsilon, \delta)\right\}_{\mathrm{span}} .
\end{gathered}
$$

REMARK 2.8. We note that $L(\varepsilon, \delta):=L_{-2} \oplus L_{-1} \oplus L_{0} \oplus L_{1} \oplus L_{2}$ is the five graded Lie (super)algebra, such that $L_{-1} \oplus L_{1}=T(\varepsilon, \delta)$ and $D(T(\varepsilon, \delta), T(\varepsilon, \delta))=$ $L_{-2} \oplus L_{0} \oplus L_{2}$ with $\left[L_{i}, L_{j}\right] \subseteq L_{i+j}$. This Lie (super)algebra construction is one of the reasons to study nonassociative algebras and triple systems.

2.2. JTSs associated with $(\varepsilon, \delta)$-FKTSs. In this section, we consider first the properties of $\{K(x, y)\}_{\text {span }}$ defined by (2.6).

Proposition 2.9. Let $U$ be an $(\varepsilon, \delta)$-FKTS. Then

$$
\begin{aligned}
K(u, v) K(x, y) & =L(v, K(x, y) u)-\delta L(u, K(x, y) v) \\
& =\varepsilon \delta L(K(u, v) y, x)-\varepsilon L(K(u, v) x, y)
\end{aligned}
$$

for any $u, v, x, y \in U$, where $L(x, y), K(x, y)$ are defined by (2.6).

PROOF. Although (2.15)-(2.16) were noted in [50] the proof was not given. Since its validity is an important tool in what follows, we give the proof below. By (2.6) it follows that

$$
\begin{aligned}
K(u, v) K(x, y) z= & K(u, v)\{x z y-\delta y z x\}=u(x z y) v-\delta v(x z y) u \\
& \quad-\delta\{u(y z x) v-\delta v(y z x) u\} \\
= & u(x z y) v-\delta v(x z y) u-\delta u(y z x) v+v(y z x) u .
\end{aligned}
$$

Next, from (2.4) we get

$$
(z x(u y v))=((z x u) y v)+\varepsilon(u(x z y) v)+(u y(z x v))
$$


as well as the relations obtained by letting $u \leftrightarrow v$, or $x \leftrightarrow y$ and so on, so that

$$
\begin{aligned}
& \varepsilon(u(x z y) v)=(z x(u y v))-((z x u) y v)-(u y(z x v)), \\
& \varepsilon(v(x z y) u)=(z x(v y u))-((z x v) y u)-(v y(z x u)), \\
& \varepsilon(u(y z x) v)=(z y(u x v))-((z y u) x v)-(u x(z y v)), \\
& \varepsilon(v(y z x) u)=(z y(v x u))-((z y v) x u)-(v x(z y u)) .
\end{aligned}
$$

Then (2.17) can be written as

$$
\begin{aligned}
\varepsilon K(u, v) & K(x, y) z \\
= & x(u y v)-(z x u) y v-u y(z x v)-\delta z x(v y u)+\delta(z x v) y u \\
& +\delta v y(z v u)-\delta z y(u x v)+\delta(z y u) x v+\delta u x(z y v) \\
& +z y(v x u)-(z y v) x u-v x(z y u) \\
= & x x(K(u, v) y)-\delta z y(K(u, v) x)-K(z x u, v) y \\
& -K(u, z x v) y-K(v, z y u) x-K(z y v, u) x \\
=K & (z, K(u, v) y) x+\delta(K(u, v) y) x z \\
& -\delta\{K(z, K(u, v) x) y+\delta(K(u, v) x) y z\}-K(z x u, v) y \\
& -K(u, z x v) y-K(v, z y u) x-K(z y v, u) x \\
=\delta( & K(u, v) y) x z-(K(u, v) x) y z \\
& +\{K(z, K(u, v) y)-K(v, z y u)-K(z y v, u)\} x \\
& -\{\delta K(z, K(u, v) x)+K(z x u, v)+K(u, z x v)\} y .
\end{aligned}
$$

Then from the definition of an $(\varepsilon, \delta)$-FKTS and (2.7) we remark that

$$
\delta K(z, K(u, v) x)+K(z x u, v)+K(u, z x v)=0,
$$

hence the last term in (2.18) vanishes. Similarly,

$$
\begin{aligned}
K(z, & K(u, v) y)-K(v, z y u)-K(z y v, u) \\
& =K(z, K(u, v) y)+\delta K(z y u, v)+\delta K(u, z y v) \\
& =\delta\{\delta K(z, K(u, v) y)+K(z y u, v)+K(u, z y v)\}=0,
\end{aligned}
$$

hence the second term in (2.18) also vanishes. Therefore, (2.18) becomes

$$
\varepsilon K(u, v) K(x, y) z=\delta(K(u, v) y) x z-(K(u, v) x) y z,
$$

which is rewritten as

$$
\varepsilon K(u, v) K(x, y)=\delta L(K(u, v) y, x)-L(K(u, v) x, y) .
$$

This proves (2.16). Next we note that

$$
[L(u, v), L(x, y)]=L(L(u, v) x, y)+\varepsilon L(x, L(v, u) y)
$$


so that, by letting $u \leftrightarrow x$ and $v \leftrightarrow y$,

$$
L(L(u, v) x, y)+\varepsilon L(x, L(v, u) y)=-L(L(x, y) u, v)-\varepsilon L(u, L(y, x) v)
$$

or

$$
L(u v x, y)+\varepsilon L(x, v u y)+L(x y u, v)+\varepsilon L(u, y x v)=0 .
$$

Letting $u \leftrightarrow x$, this also gives

$$
L(x v u, y)+\varepsilon L(u, v x y)+L(u y x, v)+\varepsilon L(x, y u v)=0 .
$$

Calculating (2.19) $-\delta(2.20)$, we obtain

$$
\begin{aligned}
& L(u v x-\delta x v u, y)+\varepsilon L(x, v u y-\delta y u v) \\
& \quad+L(x y u-\delta u y x, v)+\varepsilon L(u, y x v-\delta v x y)=0,
\end{aligned}
$$

that is,

$$
L(K(u, x) v, y)+\varepsilon L(x, K(v, y) u)+L(K(x, u) y, v)+\varepsilon L(u, K(y, v) x)=0 .
$$

Exchanging $x \leftrightarrow v$, this yields

$$
L(K(u, v) x, y)+\varepsilon L(v, K(x, y) u)+L(K(v, u) y, x)+\varepsilon L(u, K(y, x) v)=0
$$

hence, by (2.7),

$$
L(K(u, v) x, y)-\delta L(K(u, v) y, x)=-\varepsilon L(v, K(x, y) u)+\varepsilon \delta L(u, K(x, y) v),
$$

which proves the equivalence of (2.15) and (2.16) and completes the proof.

COROllary 2.10. The $K(x, y)$ defined by (2.6) satisfies a Lie relation type, that is,

$$
\begin{aligned}
& {[K(x, y),[K(u, v), K(a, b)]]} \\
& \quad=\delta K([K(u, v), K(a, b)] y, x)-K([K(u, v), K(a, b)] x, y),
\end{aligned}
$$

and the following component-wise relations are valid for $S(a, b)$ defined by (2.10):

$$
\begin{aligned}
{[K(a, b), K(x, y)] } & =\delta S(x, K(a, b) y)-\varepsilon S(K(a, b) x, y), \\
{[S(x, y), K(a, b)] } & =K(K(a, b) y, x)+\varepsilon K(K(a, b) x, y) \\
& =K(S(x, y) a, b)+K(a, S(x, y) b), \\
{[S(a, b), S(x, y)] } & =S(S(a, b) x, y)+S(x, S(a, b) y) .
\end{aligned}
$$

PROOF. We note that

$$
\begin{aligned}
& {[K(x, y),[K(u, v), K(a, b)]]} \\
& =K(x, y)(K(u, v) K(a, b)-K(a, b) K(u, v)) \\
& \quad-(K(u, v) K(a, b)-K(a, b) K(u, v)) K(x, y) \\
& =K(x, y) K(u, v) K(a, b)+K(a, b) K(u, v) K(x, y) \\
& \quad-K(x, y) K(a, b) K(u, v)-K(u, v) K(a, b) K(x, y) \\
& =K(K(a, b) K(u, v) x, y)-\delta K(K(a, b) K(u, v) y, x) \\
& \quad-K(K(u, v) K(a, b) x, y)+\delta K(K(u, v) K(a, b) y, x),
\end{aligned}
$$


by (2.28). This proves the validity of (2.21). Next, by (2.15) and (2.16), it follows that

$$
\begin{aligned}
K(a, b) K(x, y) & =L(b, K(x, y) a)-\delta L(a, K(x, y) b) \\
& =\varepsilon \delta L(K(a, b) y, x)-\varepsilon L(K(a, b) x, y) .
\end{aligned}
$$

Letting $a \leftrightarrow x$ and $b \leftrightarrow y$, then

$$
\begin{aligned}
K(x, y) K(a, b) & =L(y, K(a, b) x)-\delta L(x, K(a, b) y) \\
& =\varepsilon \delta L(K(x, y) b, a)-\varepsilon L(K(x, y) a, b),
\end{aligned}
$$

hence

$$
\begin{aligned}
& K(a, b) K(x, y)-K(x, y) K(a, b) \\
& =\delta\{L(x, K(a, b) y)+\varepsilon L(K(a, b) y, x)\} \\
& \quad-\varepsilon\{L(K(a, b) x, y)+\varepsilon L(y, K(a, b) x)\},
\end{aligned}
$$

which gives (2.22). Also, since

$$
K(K(a, b) x, y)=L(y, x) K(a, b)-\varepsilon K(a, b) L(x, y),
$$

we easily obtain (2.23). Moreover, by (2.9),

$$
K(K(a, b) y, x)=K(x y a, b)+K(a, x y b),
$$

hence

$$
K(K(a, b) y, x)+\varepsilon K(K(a, b) x, y)=K(S(x, y) a, b)+K(a, S(x, y) b) .
$$

Thus these imply that (2.23) and (2.24) hold. Straightforward calculations also show that (2.25) holds. The proof is thus complete.

REMARK 2.11. Corollary 2.10 shows that the $K(a, b)$ defined by (2.6) has an LTS structure with respect to the product

$$
[[K(a, b), K(c, d)], K(e, f)]=[K(a, b), K(c, d), K(e, f)] .
$$

Proposition 2.12. Let $U$ be an $(\varepsilon, \delta)$-FKTS. Then the $K(x, y)$ defined by $(2.6)$ satisfies a JTS relation type, that is,

$$
\begin{aligned}
& K(a, b) K(x, y) K(u, v)+K(u, v) K(x, y) K(a, b) \\
& \quad=K(K(a, b) K(x, y) u, v)-\delta K(K(a, b) K(x, y) v, u), \\
& \quad=\varepsilon \delta K(K(a, b) x, K(u, v) y)-\varepsilon K(K(a, b) y, K(u, v) x) .
\end{aligned}
$$

PROOF. From (2.15)-(2.16),

$$
\begin{aligned}
& K(u, v) K(x, y) K(a, b) \\
& \quad=L(v, K(x, y) u) K(a, b)-\delta L(u, K(x, y) v) K(a, b) \\
& \quad=\varepsilon \delta L(K(u, v) y, x) K(a, b)-\varepsilon L(K(u, v) x, y) K(a, b) .
\end{aligned}
$$


Also

$$
\begin{aligned}
K(x, y) K(u, v) & =L(y, K(u, v) x)-\delta L(x, K(u, v) y) \\
& =\varepsilon \delta L(K(x, y) v, u)-\varepsilon L(K(x, y) u, v),
\end{aligned}
$$

which becomes

$$
\begin{aligned}
& K(a, b) K(x, y) K(u, v) \\
& \quad=K(a, b) L(y, K(u, v) x)-\delta K(a, b) L(x, K(u, v) y) \\
& \quad=\varepsilon \delta K(a, b) L(K(x, y) v, u)-\varepsilon K(a, b) L(K(x, y) u, v) .
\end{aligned}
$$

Adding (2.30) and (2.31) yields

$$
\begin{aligned}
K(u, v) K(x, y) K(a, b)+K(a, b) K(x, y) K(u, v) \\
=\{L(v, K(x, y) u) K(a, b)-\varepsilon K(a, b) L(K(x, y) u, v)\} \\
\quad-\delta\{L(u, K(x, y) v) K(a, b)-\varepsilon K(a, b) L(K(x, y) v, u)\} \\
=\varepsilon \delta\{L(K(u, v) y, x) K(a, b)-\varepsilon K(a, b) L(x, K(u, v) y)\} \\
\quad-\varepsilon\{L(K(u, v) x, y) K(a, b)-\varepsilon K(a, b) L(y, K(u, v) x)\} .
\end{aligned}
$$

By (2.8), Equation (2.32) leads to (2.28) and (2.29) and completes the proof.

Let $\kappa=\{K(x, y) \mid x, y \in U\}_{\text {span }}$ and define a triple product in $\kappa$ by

$$
\left\{K_{1}, K_{2}, K_{3}\right\}:=K_{1} K_{2} K_{3}+K_{3} K_{2} K_{1} \quad\left(K_{j} \in \kappa\right) .
$$

REMARK 2.13. From Proposition 2.12 it then follows that

$\{K(a, b), K(x, y), K(c, d)\}=K(K(a, b) K(x, y) c, d)-\delta K(K(a, b) K(x, y) d, c)$.

PROPOSITION 2.14. The triple product $\left\{K_{1}, K_{2}, K_{3}\right\}$ defined by (2.33) is a JTS.

PROOF. Since the $K_{j}$ are associative the assertion follows from the last remark.

Proposition 2.15. For the triple product $\{\cdot, \cdot, \cdot\}$ defined by $(2.33)$, let $\sigma(x, y) \in$ End $\kappa$ and $\theta(x, y) \in$ End $\kappa, x, y \in U$, be defined by

$$
\begin{aligned}
\sigma(x, y) K(a, b) & :=K(K(a, b) x, y)-\varepsilon \delta K(x, K(a, b) y), \\
\theta(x, y) K(a, b) & :=K(K(a, b) x, y)+\varepsilon \delta K(x, K(a, b) y) .
\end{aligned}
$$

Then $\sigma(x, y)$ is a derivation and $\theta(x, y)$ is an anti-derivation of the JTS $\kappa$.

PROOF. We prove first that

$$
\begin{aligned}
& \sigma(x, y)\{K(a, b), K(c, d), K(e, f)\}=\{\sigma(x, y) K(a, b), K(c, d), K(e, f)\} \\
& \quad+\{K(a, b), \sigma(x, y) K(c, d), K(e, f)\}+\{K(a, b), K(c, d), \sigma(x, y) K(e, f)\} .
\end{aligned}
$$


Indeed, by the properties of $K(x, y)$, we calculate:

$$
\begin{aligned}
& \sigma(x, y)\{K(a, b), K(c, d), K(e, f)\} \\
& =\sigma(x, y)(K(K(a, b) K(c, d) e, f)+K(e, K(a, b) K(c, d) f)) \\
& =K(K(K(a, b) K(c, d) e, f) x, y)-\varepsilon \delta K(x, K(K(a, b) K(c, d) e, f) y) \\
& +K(K(e, K(a, b) K(c, d) f) x, y)-\varepsilon \delta K(x, K(e, K(a, b) K(c, d) f) y) \\
& =K(\{K(a, b), K(c, d), K(e, f)\} x, y) \\
& -\varepsilon \delta K(x,\{K(a, b), K(c, d), K(e, f)\} y), \\
& \{\sigma(x, y) K(a, b), K(c, d), K(e, f)\} \\
& =\{(K(K(a, b) x, y)-\varepsilon \delta K(x, K(a, b) y)), K(c, d), K(e, f)\} \\
& =\{K(e, f), K(c, d), K(K(a, b) x, y)\} \\
& -\varepsilon \delta\{K(e, f), K(c, d), K(x, K(a, b) y)\} \\
& =K(K(e, f) K(c, d) K(a, b) x, y)+K(K(a, b) x, K(e, f) K(c, d) y) \\
& -\varepsilon \delta K(K(e, f) K(c, d) x, K(a, b) y) \\
& -\varepsilon \delta K(x, K(e, f) K(c, d) K(a, b) y), \\
& \{K(a, b), \sigma(x, y) K(c, d), K(e, f)\} \\
& =\{K(a, b), K(K(c, d) x, y), K(e, f)\} \\
& -\varepsilon \delta\{K(a, b), K(x, K(c, d) y), K(e, f)\} \\
& =\varepsilon \delta K(K(a, b) K(c, d) x, K(e, f) y)+\varepsilon \delta K(K(e, f) K(c, d) x, K(a, b) y) \\
& -K(K(a, b) x, K(e, f) K(c, d) y)-K(K(e, f) x, K(a, b) K(c, d) y), \\
& \{K(a, b), K(c, d), \sigma(x, y) K(e, f)\} \\
& =\{K(a, b), K(c, d), K(K(e, f) x, y)\} \\
& -\varepsilon \delta\{K(a, b), K(c, d), K(x, K(e, f) y)\} \\
& =K(K(a, b) K(c, d) K(e, f) x, y)+K(K(e, f) x, K(a, b) K(c, d) y) \\
& -\varepsilon \delta K(K(a, b) K(c, d) x, K(e, f) y) \\
& -\varepsilon \delta K(x, K(a, b) K(c, d) K(e, f) y) \text {. }
\end{aligned}
$$

Thus, these mean that $\sigma(x, y)$ is a derivation of $\kappa$. Similarly, we can prove that $\theta(x, y)$ is an anti-derivation, but we omit this here. This completes the proof.

An $(\varepsilon, \delta)$-FKTS $U$ is called unitary if the identity map Id is contained in $\kappa:=$ $K(U, U)$, that is, if there exist $a_{i}, b_{i} \in U$ such that

$$
\sum_{i} K\left(a_{i}, b_{i}\right)=\mathrm{Id}
$$

REMARK 2.16. We note that a balanced triple system (that is, which satisfies $K(x, y)=\langle x \mid y\rangle \mathrm{Id})$ is unitary, since $\operatorname{Id} \in \kappa=K(U, U)$. If we assume the unitary property, we can get more interesting results as follows. 
Proposition 2.17. Let $U$ be an $(\varepsilon, \delta)$-FKTS. If $U$ is unitary, then:

(i)

$$
\varepsilon=\delta \quad(\text { or } \varepsilon \delta=1)
$$

(ii)

$$
K(x, y)=L(y, x)-\varepsilon L(x, y)=-\varepsilon A(x, y)
$$

(iii)

$$
\begin{aligned}
& K(x, y) K(u, v)+K(u, v) K(x, y) \\
& \quad=K(K(u, v) x, y)+K(x, K(u, v) y) \\
& =K(u, K(x, y) v)+K(K(x, y) u, v)
\end{aligned}
$$

(iv) $K(x, y)$ is an anti-derivation of $U$.

PROOF. From Proposition 2.9,

$$
\begin{aligned}
K(u, v) K(x, y) & =L(v, K(x, y) u)-\delta L(u, K(x, y) v) \\
& =\varepsilon \delta L(K(u, v) y, x)-\varepsilon L(K(u, v) x, y) .
\end{aligned}
$$

Choosing $u=a_{i}$ and $v=b_{i}$ and summing over $i$ leads, by (2.36), to

$$
K(x, y)=\varepsilon \delta L(y, x)-\varepsilon L(x, y) .
$$

Now, setting $x=a_{i}$ and $y=b_{i}$, gives

$$
K(u, v)=L(v, u)-\delta L(u, v) .
$$

Changing $u \rightarrow x$ and $v \rightarrow y$ in (2.38) and comparing it with (2.37) requires the validity of

$$
(\varepsilon \delta-1) L(y, x)=(\varepsilon-\delta) L(x, y) .
$$

If $\varepsilon \delta=1$ then $\varepsilon=\delta$ and (i) is clear. Moreover, if $\varepsilon \delta=-1$ then $\varepsilon=-\delta$ and the last identity gives $-2 L(y, x)=2 \varepsilon L(x, y)$, that is, $L(y, x)=-\varepsilon L(x, y)$. Thus by (2.37), $K(x, y)=-L(y, x)-\varepsilon L(x, y)=0$ which contradicts (2.36). This proves $\varepsilon=\delta$. Then, by (2.37), it follows that $K(x, y)=L(y, x)-\varepsilon L(x, y)=-\varepsilon A(x, y)$, which is (ii), and so $K(x, y)$ is an anti-derivation of $U$.

Next, Equations (2.15)-(2.16) are rewritten as

$$
\begin{aligned}
K(u, v) K(x, y) & =L(v, K(x, y) u)-\varepsilon L(u, K(x, y) v) \\
& =L(K(u, v) y, x)-\varepsilon L(K(u, v) x, y)
\end{aligned}
$$

so that

$$
\begin{aligned}
K(x, y) K(u, v) & =L(y, K(u, v) x)-\varepsilon L(x, K(u, v) y) \\
& =L(K(x, y) v, u)-\varepsilon L(K(x, y) u, v)
\end{aligned}
$$


and hence, by (ii),

$$
\begin{aligned}
K(u, v) K(x, y)+K(x, y) K(u, v)= & L(K(u, v) y, x)-\varepsilon L(K(u, v) x, y) \\
& +L(y, K(u, v) x)-\varepsilon L(x, K(u, v) y) \\
= & K(x, K(u, v) y)+K(K(u, v) x, y) .
\end{aligned}
$$

Proposition 2.18 (Associated Jordan algebra). Let $U$ be a unitary $(\varepsilon, \delta)-F K T S$. Then the commutative product in $K(U, U)$ defined by

$$
\begin{aligned}
K(u, v) * K(x, y) & =K(u, v) K(x, y)+K(x, y) K(u, v) \\
& =K(x, K(u, v) y)+K(K(u, v) x, y)
\end{aligned}
$$

defines a Jordan algebra $\kappa^{*}$. Moreover, $\sigma(x, y)$ is a derivation of the Jordan algebra $\kappa^{*}$, that is,

$$
\begin{array}{r}
\sigma(a, b)(K(u, v) * K(x, y))=(\sigma(a, b) K(u, v)) * K(x, y) \\
+K(u, v) *(\sigma(a, b) K(x, y)) .
\end{array}
$$

Proof. By (2.34) we have $\sigma(x, y) \mathrm{Id}=0$ since $\varepsilon \delta=1$. Applying Proposition 2.15 to

$$
\begin{aligned}
& \sigma(a, b)\{K(u, v), \mathrm{Id}, K(x, y)\}=\{\sigma(a, b) K(u, v), \mathrm{Id}, K(x, y)\} \\
& \quad+\{K(u, v), \sigma(a, b) \mathrm{Id}, K(x, y)\}+\{K(u, v), \mathrm{Id}, \sigma(a, b) K(x, y)\}
\end{aligned}
$$

gives (2.40), since $\{K(u, v), \mathrm{Id}, K(x, y)\}=K(u, v) * K(x, y)$. This completes the proof.

REMARK 2.19. We note that the property of $\kappa$ is the same as the property of

$$
\mathcal{L}_{-2}=\left\{\left(\begin{array}{cc}
0 & K(x, y) \\
0 & 0
\end{array}\right) \mid x, y \in U(\varepsilon, \delta)\right\}_{\text {span }},
$$

thus the investigation of $U(\varepsilon, \delta)$ means the study of the standard embedding Lie (super)algebra.

REMARK 2.20. We give below another proof of the facts that $\sigma(x, y)$ is a derivation of $\kappa$ and $\theta(x, y)$ is an anti-derivation, since the method may be used for some structure theory of triple systems to be given elsewhere.

(i) Note that $\sigma(x, y)=\varepsilon$ ad $S(x, y)$ because, according to (2.23),

$$
\begin{aligned}
\varepsilon[S(x, y), K(a, b)] & =\varepsilon K(K(a, b) y, x)+K(K(a, b) x, y) \\
& =-\varepsilon \delta K(x, K(a, b) y)+K(K(a, b) x, y) \\
& =\sigma(x, y) K(x, y) .
\end{aligned}
$$

Hence, it is clear that $\sigma(x, y)$ is a derivation of the JTS without any calculation, since ad $S$ is a derivation of any associative algebra. 
(ii) Similarly, by (2.10),

$$
\begin{aligned}
A(x, y) K(a, b)+K(a, b) A(x, y) & =K(A(x, y) a, b)+K(a, A(x, y) b) \\
& =-\delta K(x, K(a, b) y)-\varepsilon K(K(a, b) x, y) .
\end{aligned}
$$

On the other hand, by (2.35),

$$
\theta(x, y)(K(a, b))=K(K(a, b) x, y)+\varepsilon \delta K(x, K(a, b) y) .
$$

Thus this means that $\theta(x, y)(K(a, b))=-\varepsilon\{A(x, y), K(a, b)\}$, where $\{A, B\}:=$ $A B+B A$. Then the map $\theta(x, y)$ is an anti-derivation of the JTS $\kappa$ induced from $U$. These imply that

$$
\begin{aligned}
A(x, y)\{ & K(a, b), K(c, d), K(e, f)\}+\{K(a, b), K(c, d), K(e, f)\} A(x, y) \\
=A(x, y) K(K(a, b) K(c, d) e, f)+K(K(a, b) K(c, d) e, f) A(x, y) & \\
& \quad-\delta(A(x, y) K(K(a, b) K(c, d) f, e)+K(K(a, b) K(c, d) f, e) A(x, y))
\end{aligned}
$$

from the fact that any associative algebra satisfies a relation

$$
A(B C D)+(B C D) A=(A B+B A) C D-B(A C+C A) D+B C(A D+D A) .
$$

2.3. Simplicity. In this section, we will consider unitary $(-1,-1)$-FKTSs $U$ unless specified otherwise and the JTSs $\kappa$ induced from $U$.

LEMMA 2.21. If $U$ is a simple unitary $(\delta, \delta)$-FKTS, then $\kappa$ has no nontrivial proper Der $\kappa$-invariant ideal.

PROOF. Since the proof is essentially the same for both cases $\delta=1$ and $\delta=-1$, we will consider here only the case of $\delta=-1$.

Let $\beta \neq \kappa$ be a Der $\kappa$-invariant ideal of $\kappa$ and $\sum_{i} K\left(s_{i}, t_{i}\right)$ be an arbitrary element of $\beta$. Since $\sigma(x, y)$, which maps

$$
\sum_{i} K\left(s_{i}, t_{i}\right) \rightarrow \sum_{i}\left(K\left(K\left(s_{i}, t_{i}\right) x, y\right)-K\left(x, K\left(s_{i}, t_{i}\right) y\right)\right),
$$

is a derivation of $\kappa$, then

$$
\sum_{i}\left(K\left(K\left(s_{i}, t_{i}\right) x, y\right)-K\left(x, K\left(s_{i}, t_{i}\right) y\right)\right) \in \beta,
$$

for all $\sum_{i} K\left(s_{i}, t_{i}\right) \in \beta, x, y \in U$. On the other hand, since Id $\in \kappa$, we get

$$
\left\{\sum_{i} K\left(s_{i}, t_{i}\right), \mathrm{Id}, K(x, y)\right\}=\sum_{i}\left(K\left(K\left(s_{i}, t_{i}\right) x, y\right)+K\left(x, K\left(s_{i}, t_{i}\right) y\right)\right) .
$$

From the fact that $\sum_{i} K\left(s_{i}, t_{i}\right) \in \beta, \operatorname{Id} \in \kappa$ and $\beta$ is an ideal, thus we obtain

$$
\sum_{i}\left(K\left(K\left(s_{i}, t_{i}\right) x, y\right)+K\left(x, K\left(s_{i}, t_{i}\right) y\right)\right) \in \beta .
$$


Hence, by (2.41)-(2.42), it follows that $\sum_{i} K\left(K\left(s_{i}, t_{i}\right) x, y\right) \in \beta$. This implies that $K(\beta U, U) \subset \beta$.

We set

$$
V:=\{x \in U \mid K(x, U) \subset \beta\} .
$$

Then $\beta U \subset V$ holds.

By Proposition 2.17, $K(a, b)=L(a, b)+L(b, a)$ and by (2.9) we can easily show that $V$ is an ideal of $U$ as follows. Indeed, by (2.43), we note that $K(V, U) \subset \beta$, so that $K(V, U) U \subset \beta U \subset V$. Hence $K(U, K(V, U) U) \subset \beta$, that is, for all $c \in V$, $a, b, d \in U$, we get

$$
K(a, K(c, d) b) \in \beta .
$$

By (2.9),

$$
K(a b c, d)=-K(c, a b d)+K(a, K(c, d) b)
$$

and then from

$$
K(c, a b d) \in K(V, U) \subset \beta, \quad K(a, K(c, d) b) \in \beta
$$

it follows that $K(a b c, d) \in \beta$, hence $a b c \in V$, that is, for all $c \in V$ implies $a b c \in V$, for all $a, b \in U$.

Also, we get

$$
c b a+a b c=K(a, c) b \in K(U, V) U \subset V
$$

and thus

$$
c b a \in V \quad \text { since } a b c \in V, c \in V, a, b \in U \text {. }
$$

Again, by (ii),

$$
a c b+c a b=K(a, c) b \in K(U, V) U \subset V .
$$

But, for all $c \in V$ and $a, b \in U$, we had $c b a \in V$, in particular, $c a b \in V$ and summing, $a c b \in V$. Therefore we get

$$
(U U V) \subset V,(U V U) \subset V,(V U U) \subset V .
$$

That is, $V$ is an ideal of $U$. Since $U$ is simple, either $U=V$ or $V=\{0\}$. The case of $V=U$ contradicts the assumption that $K(x, U) \subset \beta \neq \kappa$ for all $x \in V$. It must be that $V=\{0\}$. Hence $\beta U=\{0\}$. This implies $\beta=\{0\}$, which completes the proof.

PROPOSITION 2.22. Let $U$ be a unitary $(-1,-1)$-FKTS over a field of characteristic 0 and $\kappa$ be the special JTS associated with $U$. If $U$ is simple, then $\kappa$ is semisimple.

PROOF. Let $R(\kappa)$ be the radical of the JTS $\kappa$. It is well known that $R(\kappa)$ is a derivation-invariant ideal of $\kappa$. By Lemma 2.21, we have $R(\kappa)=0$ or $R(\kappa)=\kappa$. But the case of $R(\kappa)=\kappa$ contradicts the hypothesis of unitarity. This completes the proof. 
Lemma 2.23. Let $\kappa$ be as in Proposition 2.22. If $\kappa$ is semisimple, then any ideal of $\kappa$ is Der $\kappa$ invariant as well as Anti-Der $\kappa$ invariant.

PROOF. Assume that $\kappa$ is semisimple. Then for a JTS it is well known that Der $\kappa=$ Inn Der $\kappa$.

From the fact that the set of the inner derivations of $\kappa$ is the linear span of the set $\{L(x, y)-L(y, x) \mid x, y \in \kappa\}$, it follows that any ideal of $\kappa$ is Der $\kappa$-invariant.

Indeed, if $\beta \triangleleft \kappa$ and $z \in \beta,(L(a, b)-L(b, a)) z=a b z-b a z \in \beta$, hence $D z \in \beta$ for any $D$ inner derivation, and hence for any derivation.

The case of anti-derivation is similarly straightforward, because the set of antiderivations is the linear span of $\{L(a, b)+L(b, a)\}$. This completes the proof.

The JTS $\kappa$ is called nondegenerate if $K(x, y)=0$ for all $y \in U$ implies $x=0$.

THEOREM 2.24. Let $U$ be a unitary $(-1,-1)$-FKTS over a field of characteristic 0 and $\kappa$ be the special JTS associated with $U$. Then the following are equivalent:

(i) $U$ is simple;

(ii) $\kappa$ is simple and nondegenerate.

PROOF. We first prove that (i) implies (ii). From Lemmas 2.21 and 2.23 it follows that $\kappa$ is simple. If $\kappa$ is degenerate, then we can show that $V=\{x \in U \mid K(x, U)=0\}$ is a nonzero ideal of $U$ by means of the relations $K(c, d)=L(c, d)+L(d, c)$ and (2.9).

Indeed, take $a \in V, b, c, x, y \in U$. From (2.9),

$$
K(K(a, b) x, y)-K(y x a, b)-K(a, y x b)=0 .
$$

Then it follows by $K(a, y x b)=0$ and $K(K(a, b) x, y)=0$ that $K(y x a, b)=0$ and hence

$$
y x a \in V .
$$

Now from $K(a, b) c=a c b+b c a$, it follows that

$$
a c b \in V
$$

since $K(a, b)=0$ and $b c a \in V$. Finally, from $K(a, b) c=a b c+b a c$ we obtain

$$
\text { bac } \in V
$$

since $K(a, b)=0, a b c \in V$. Thus $V$ is an ideal of $U$.

Since $U$ is simple and $V \neq\{0\}$ we have that $V=U$. Therefore we get $K(U, U)=0$, which contradicts the unitary hypothesis. Hence, $\kappa$ is nondegenerate.

To prove that (ii) implies (i), let $V \neq\{0\}$ be an ideal of $U$. We set

$$
\mathbf{M}:=\{K(x, y) \mid x \in V, y \in U\}_{\text {span }} .
$$

Then by the results of Propositions 2.9 and 2.12, we can show that $\mathbf{M}$ is an ideal of the JTS $\kappa$. 
Indeed, we see that for all $K(x, y) \in \mathbf{M}, x \in V, y \in U$,

$$
\{K(a, b), K(c, d), K(x, y)\}=K(K(a, b) K(c, d) x, y)+K(K(a, b) K(c, d) y, x)
$$

and so

$$
K(K(a, b) K(c, d) x, y) \in \mathbf{M}
$$

From

$$
K(K(a, b) K(c, d) x, y)+K(K(a, b) K(c, d) y, x) \in K(V, U),
$$

it follows that

$$
\{K(a, b), K(c, d), K(x, y)\} \in K(V, U)=\mathbf{M} .
$$

Next, from $K(a, b) x \in V, K(c, d) x \in V$,

$$
\begin{aligned}
& \{K(a, b), K(x, y), K(c, d)\} \\
& \quad=K(K(a, b) x, K(c, d) y)+K(K(c, d) x, K(a, b) y) \in K(V, U)=\mathbf{M} .
\end{aligned}
$$

That is, $\{K(a, b), K(x, y), K(c, d)\} \in \mathbf{M}$, thus $\mathbf{M}$ is an ideal of $\kappa$. Since $\kappa$ is simple and nondegenerate, the above fact gives $\mathbf{M}=\kappa$.

Observe now that $K(x, y) U \subset V$ for any $x \in V$ and $y \in U$. Then $\mathbf{M} U \subset V$. Since $\mathbf{M}=\kappa$, we get $\kappa U \subset V$. Since $U$ is unitary (that is, Id $\in \kappa$ ), this means that $V=U$. This completes the proof.

REMARK 2.25. For $(1,1)-F K T S s$ we refer to [15].

2.4. Examples. In this section we will give several examples of $(-1,-1)$-FKTSs. Let $\mathcal{M}_{m, n}(\Phi)$ denote the vector space of $m \times n$ matrices over $\Phi$ and for $x \in \mathcal{M}_{m, n}(\Phi)$ denote by $x^{\top}$ the transposed matrix.

THEOREM 2.26. Let $U$ be the set $\mathcal{M}_{k, n}(\Phi)$. Then $U$ is a unitary $(-1,-1)$-FKTS with respect to the product

$$
x y z:=z y^{\top} x+y x^{\top} z-x y^{\top} z, \quad x, y, z \in \mathcal{M}_{k, n}(\Phi) .
$$

Furthermore, this triple system is simple.

Proof. From $\kappa=\left\{x^{\top} z+z^{\top} x\right\}_{\text {span }}=\left\{A \mid A^{\top}=A, A \in \mathcal{M}_{n, n}(\Phi)\right\}$, by means of Theorem 2.24, straightforward calculations show that the triple system $U$ is simple and unitary.

REMARK 2.27. Hence by the methods of the standard embedding associated with $U$ (see Section 1 in this paper or [26]), we can obtain the standard embedding Lie superalgebra as follows:

(i) $L(U)=D(m, n)$, if $k=2 m(m \geq 1)$;

(ii) $L(U)=B(m, n)$, if $k=2 m+1(m \geq 0)$. 
EXAMPLE $2.28[6,26]$. Let $U$ be a balanced $(-1,-1)$-FKTS associated with quaternion (octonion) algebra $\mathbf{H}(\mathbf{O})$. Then we have the construction of simple Lie superalgebras $D(2,1: \alpha), F(4)$ and $G(3)$ by the method of the standard embedding. Indeed, since $K(x, y)=\langle x \mid y\rangle \mathrm{Id}$, it is clear that $\kappa=\{K(x, y)\}_{\text {span }}$ is one-dimensional and so nondegenerate, hence simple.

EXAMPlE 2.29 (Counterexample [28]). For the Lie superalgebras $P(n), Q(n)$ we have a construction from the cases of anti-JTSs. This implies a nonunitary case of $(-1,-1)$-FKTSs, since $K(x, y)$ is identically zero.

EXAMPLE 2.30. Let $A$ be an involutive associative algebra so that $(x y) z=x(y z)$. Then both $x y z:=(x \bar{y}) z-(z \bar{y}) x+(z \bar{x}) y$ and $x \circ y \circ z:=\overline{\bar{x}} \bar{y} \bar{z}=z(\bar{y} x)-x(\bar{y} z)+$ $y(\bar{x} z)$ are $(-1,-1)$-FKTSs. Moreover, if there exists a $f \in A$ satisfying $f \bar{f}=e=$ identity element of $A$, then this $(-1,-1)$-FKTS is unitary.

Let $\gamma$ be the trace form of $(-1,-1)$-FKTS given by

$$
\gamma(x, y):=\frac{1}{2} \operatorname{Tr}[2(R(x, y)+R(y, x))+L(x, y)+L(y, x)]
$$

where $\operatorname{Tr}$ denotes the trace and let us calculate it for our previous examples.

In the case of Theorem 2.26, straightforward calculations give $\gamma(x, y)=c_{x, y}(2 n+$ $2-k$ ) for some $c_{x, y} \in \Phi$.

In the case of $D(2,1 ; \alpha)$, we obtain $\gamma(x, y)=(4-N)\langle x \mid y\rangle$, for $N=\operatorname{dim} U$, and $K(x, y)=\langle x \mid y\rangle$ Id.

REMARK 2.31. By [16], the Killing form $\alpha(t, s)$ of an anti-LTS is given by

$$
\alpha(t, s)=\frac{1}{2} \operatorname{Tr}(R(t, s)-R(s, t)),
$$

where $R(x, y) z=[z x y]$. We note that this formula is a variation of the case of an LTS trace form (= Killing form) $\alpha(t, s)$ defined by

$$
\alpha(t, s)=\frac{1}{2} \operatorname{Tr}(R(t, s)+R(s, t)) .
$$

Proposition 2.32 [16]. For the Killing forms (= bilinear trace forms) of the $(-1,-1)-F K T S$, the anti-LTS, and the Lie superalgebra:

(i) $\quad \alpha\left(\left(\begin{array}{c}a \\ b\end{array}\right),\left(\begin{array}{l}c \\ d\end{array}\right)\right)=\gamma(c, b)-\gamma(d, a)$;

(ii) $\alpha(x, y)=\beta(y, x)$,

where $\beta(x, y)$ is the Killing form of the standard embedding Lie superalgebra associated with their triple systems.

REMARK 2.33. For $k=2(n+1)$, that is, $m=n+1$ in Theorem 2.26, from the above results, the standard embedding Lie superalgebra $D(n+1, n)$ is degenerate. For $N=4$ in the first example, similarly, $D(2,1 ; \alpha)$ is degenerate.

REMARK 2.34. Let $U$ be a $(-1,-1)$-FKTS and $\gamma: U \times U \rightarrow \Phi$ be the trace form given by (2.46). If $\gamma$ is nondegenerate, then $U$ is a direct sum of simple ideals (to be shown elsewhere). 


\section{3. $\delta$-structurable algebras}

The motivation for the study of such nonassociative algebras is as follows. The existence of the class of nonassociative algebras called structurable algebras is an important generalization of Jordan algebras giving a construction of Lie algebras. Hence from our concept, by means of triple products, we define a generalization of such class to construct Lie superalgebras as well as Lie algebras.

Our start point briefly described in a historical setting is the construction of Lie (super)algebras starting from a class of nonassociative algebras. Hence within the general framework of $(\epsilon, \delta)$-FKTSs $(\epsilon, \delta= \pm 1)$ and the standard embedding Lie (super)algebra construction studied in $[6,7,13-15,28]$ (see also references therein) we define $\delta$-structurable algebras as a class of nonassociative algebras with involution which coincides with the class of structurable algebras for $\delta=1$ as introduced and studied in $[1,2]$. Structurable algebras are a class of nonassociative algebras with involution that include Jordan algebras (with trivial involution), associative algebras with involution, and alternative algebras with involution. They are related to GJTSs of second order (or $(-1,1)$-FKTSs) as introduced and studied in [31, 32] and further studied in [3, 4, 30, 39-42, 45] (see also references therein). Their importance lies with constructions of five graded Lie algebras

$$
L(\epsilon, \delta)=L_{-2} \oplus L_{-1} \oplus L_{0} \oplus L_{1} \oplus L_{2}, \quad\left[L_{i}, L_{j}\right] \subseteq L_{i+j}
$$

For $\delta=-1$ the anti-structurable algebras defined here are a new class of nonassociative algebras that may similarly shed light on the notion of $(-1,-1)$-FKTSs, hence (by $[6,7]$ ) on the construction of Lie superalgebras and Jordan algebras as will be shown.

Let $\left(\mathcal{A},{ }^{-}\right)$be a finite-dimensional nonassociative unital algebra with involution (involutive anti-automorphism, that is, $\overline{\bar{x}}=x, \overline{x y}=\bar{y} \bar{x}, x, y \in \mathcal{A}$ ) over $\Phi$. The identity element of $\mathcal{A}$ is denoted by 1 . Since char $\Phi \neq 2$, by [1] we have $\mathcal{A}=\mathcal{H} \oplus \mathcal{S}$, where $\mathcal{H}=\{a \in \mathcal{A} \mid \bar{a}=a\}$ and $\mathcal{S}=\{a \in \mathcal{A} \mid \bar{a}=-a\}$.

Suppose that $x, y, z \in \mathcal{A}$. Put $[x, y]:=x y-y x$ and $[x, y, z]:=(x y) z-x(y z)$. Note that

$$
\overline{[x, y, z]}=-[\bar{z}, \bar{y}, \bar{x}] .
$$

The operators $L_{x}$ and $R_{x}$ are defined by $L_{x}(y):=x y, R_{x}(y):=y x$.

For $\delta= \pm 1$ and $x, y \in \mathcal{A}$ define

$$
\begin{gathered}
{ }^{\delta} V_{x, y}:=L_{L_{x}(\bar{y})}+\delta\left(R_{x} R_{\bar{y}}-R_{y} R_{\bar{x}}\right), \\
{ }^{\delta} B_{\mathcal{A}}(x, y, z):={ }^{\delta} V_{x, y}(z)=(x \bar{y}) z+\delta[(z \bar{y}) x-(z \bar{x}) y], \quad x, y, z \in \mathcal{A} .
\end{gathered}
$$

${ }^{+} B_{\mathcal{A}}(x, y, z)$ is called the triple system obtained from the algebra $\left(\mathcal{A},{ }^{-}\right)$. We will call

${ }^{-} B_{\mathcal{A}}(x, y, z)$ the anti-triple system obtained from the algebra $\left(\mathcal{A},{ }^{-}\right)$. We shall write

$$
V_{x, y}:={ }^{\delta} V_{x, y}, \quad B_{\mathcal{A}}:=\left({ }^{\delta} B_{\mathcal{A}}, \mathcal{A}\right) .
$$


REMARK 3.1. The upper left index notation is chosen in order to avoid confusion with the upper right index notation of [1] which has a different meaning.

A unital nonassociative algebra with involution $\left(\mathcal{A},{ }^{-}\right)$is called a structurable algebra if the identity

$$
\left[V_{u, v}, V_{x, y}\right]=V_{V_{u, v}(x), y}-V_{x, V_{v, u}(y)}
$$

is satisfied for $V_{u, v}={ }^{+} V_{u, v}, V_{x, y}={ }^{+} V_{x, y}, u, v, x, y \in \mathcal{A}$, and we will call $\left(\mathcal{A},{ }^{-}\right)$an anti-structurable algebra if the identity (3.6) is satisfied for $V_{u, v}={ }^{-} V_{u, v}, V_{x, y}=$ $-V_{x, y}$.

If $\left(\mathcal{A},{ }^{-}\right)$is structurable then, in the terminology of [32], the triple system $B_{\mathcal{A}}$ is called a generalized Jordan triple system and by [8], $B_{\mathcal{A}}$ is a GJTS of second order, that is, satisfies the identities (2.4) and (2.5). If $\left(\mathcal{A},{ }^{-}\right)$is anti-structurable then we call $B_{\mathcal{A}}$ an anti-GJTS.

Put $T_{x}:=V_{x, 1}$ for $x \in \mathcal{A}$. Then, by (3.3),

$$
T_{x}=L_{x}+\delta R_{x-\bar{x}}
$$

for $x \in \mathcal{A}$. In particular, $T_{h}=L_{h}$ for $h \in \mathcal{H}$.

REMARKS. (i) If $u=h \in \mathcal{H}$ and $x, y \in \mathcal{A}$, (3.6) becomes

$$
\left[L_{h}, V_{x, y}\right]=V_{h x, y}-V_{x, h y} .
$$

Identity (3.8) written in element form is

$$
\begin{gathered}
((h x) \bar{y}) z-h((x \bar{y}) z)+\delta[((h z) \bar{y}) x-h((z \bar{y}) x)-((h z) \bar{x}) y+h((z \bar{x}) y)] \\
=(x(\bar{y} h)) z-(x \bar{y})(h z)+\delta[(z(\bar{y} h)) x \\
-(z \bar{y})(h x)+(z(\bar{x} h)) y-(z \bar{x})(h y)]
\end{gathered}
$$

for $x, y, z \in \mathcal{A}$.

(ii) Suppose that ${ }^{-}$is the identity map and hence that $\mathcal{A}$ is commutative. If $\left(\mathcal{A},{ }^{-}\right)$ is $\delta$-structurable then $\mathcal{A}$ is a Jordan algebra, by [22]. Conversely, by [36, Section 3], any Jordan algebra satisfies (3.8) if $V_{x, y}={ }^{+} V_{x, y}$ for $x, y \in \mathcal{A}$, hence it is structurable. Thus, by (3.9), any Jordan algebra is anti-structurable if it satisfies

$$
((h x) y) z-h((x y) z)=(x(y h)) z-(x y)(h z)
$$

for $h, x, y, z \in \mathcal{A}$. Using commutativity, then (3.10) for example can be written $[x, h, y] z=[x y, z, h]$. Clearly, (3.10) is satisfied by an associative algebra.

(iii) If $x \in \mathcal{A}$ and $T_{x}(1)=0$ then $x=0$, by [22].

For $s \in \mathcal{S}$ and $h \in \mathcal{H}$ we say that $\left(\mathcal{A},{ }^{-}\right)$is $\mathcal{S}$ skew-alternative if $[s, x, y]=$ $-[x, s, y]$ while $\left(\mathcal{A},{ }^{-}\right)$is $\mathcal{H}$ skew-alternative if $[h, x, y]=-[x, h, y]$ for $x, y \in \mathcal{A}$. We remark that if $\left(\mathcal{A},{ }^{-}\right)$is $\mathcal{S}$ skew-alternative then by [1, Section 1],

$$
[s, x, y]=-[x, s, y]=[x, y, s], \quad s \in \mathcal{S}, x, y \in \mathcal{A},
$$

while if $\left(\mathcal{A},{ }^{-}\right)$is $\mathcal{H}$ skew-alternative then by (3.2),

$$
[h, x, y]=-[x, h, y]=[x, y, h], \quad h \in \mathcal{H}, x, y \in \mathcal{A} .
$$


Proposition 3.2 [22]. If $\left(\mathcal{A},{ }^{-}\right)$is structurable, then $\left(\mathcal{A},{ }^{-}\right)$is $\mathcal{S}$ skew-alternative. If $\left(\mathcal{A},{ }^{-}\right)$is anti-structurable, then $\left(\mathcal{A},{ }^{-}\right)$is $\mathcal{H}$ skew-alternative.

REMARKS. (i) If $(\mathcal{A},-)$ is anti-structurable then (3.12) is valid symmetrically with respect to $x$ and $y$, by [22].

(ii) Let $\left(\mathcal{A},{ }^{-}\right)$be a $\delta$-structurable algebra and let $\operatorname{Der}\left(\mathcal{A},{ }^{-}\right)$be the set of derivations of $\mathcal{A}$ that commute with ${ }^{-}$. By $\operatorname{Remark}$ (iii) above $T_{\mathcal{A}} \cap \operatorname{Der}\left(\mathcal{A},{ }^{-}\right)=0$ and so we may define the structure algebra $\operatorname{Str}\left(\mathcal{A},{ }^{-}\right):=T_{\mathcal{A}} \oplus \operatorname{Der}\left(\mathcal{A},{ }^{-}\right)$. This algebra plays an important role in the structure study of structurable algebras [1] and may play a role in the structure study of anti-structurable algebras (theory to be presented elsewhere).

3.1. Examples. For examples of structurable algebras we refer to $[1,2]$.

REMARK 3.3. Let $(B, U)$ and $\left(B^{\prime}, U^{\prime}\right)$ be two triple systems. We say that a linear map $\mu$ of $U$ into $U^{\prime}$ is a homomorphism if $\mu$ satisfies $\mu(B(x, y, z))=$ $B^{\prime}(\mu(x), \mu(y), \mu(z)), x, y, z \in U$. Moreover, if $\mu$ is bijective, then $\mu$ is called an isomorphism. In this case $(B, U)$ and $\left(B^{\prime}, U^{\prime}\right)$ are said to be isomorphic.

Let $\left(A,^{-}\right)$be a unital nonassociative algebra over $\Phi$ with involution ${ }^{-}$and let $\left(A^{o p},^{-}\right)$denote the opposite algebra, that is, the algebra with multiplication defined by $x ._{o p} y=y x, x, y \in A$, where in the right-hand side of the equality the multiplication is done in $A$. The algebras $\left(A,^{-}\right)$and $\left(A^{o p},^{-}\right)$are isomorphic under the map $x \mapsto \bar{x}$ (this is true for any algebra with involution). Let us define

$$
\begin{gathered}
{ }^{\delta} V_{x, y}^{o p}:=R_{R_{x}(\bar{y})}+\delta\left(L_{x} L_{\bar{y}}-L_{y} L_{\bar{x}}\right), \\
{ }^{\delta} B_{\mathcal{A}}^{o p}(x, y, z):={ }^{\delta} V_{x, y}^{o p}(z)=z(\bar{y} x)+\delta[x(\bar{y} z)-y(\bar{x} z)], \quad x, y, z \in \mathcal{A} .
\end{gathered}
$$

Then $\mathcal{A}$ is a $\delta$-structurable algebra if and only if $\mathcal{A}^{o p}$ is a $\delta$-structurable algebra since clearly, $B_{A}^{o p}$ is the triple system obtained from the algebra $\left(\mathcal{A}^{o p}{ }^{-}\right)$, and so $B_{\mathcal{A}}$ and $B_{\mathcal{A}}^{o p}$ are isomorphic under the map $x \mapsto \bar{x}$, by (3.4) and (3.14).

EXAMPLES. Let $\mathcal{M}_{m, n}(\Phi)$ denote the vector space of $m \times n$ matrices over $\Phi$ and for $x \in \mathcal{M}_{m, n}(\Phi)$ denote by $x^{\top}$ the transposed matrix.

(i) $\mathcal{M}_{m, n}(\Phi)$ with the product

$$
\{x, y, z\}:=x y^{\top} z+\delta\left(z y^{\top} x-z x^{\top} y\right),
$$

where $x, y, z \in \mathcal{M}_{m, n}(\Phi)$, is a $(-1, \delta)$-FKTS. Indeed, straightforward calculations show that (2.4) and (2.5) hold. Hence $\mathcal{M}_{n, n}(\Phi)$ with the involution $x \mapsto x^{\top}$ is a $\delta$-structurable algebra.

(ii) $\mathcal{M}_{m, n}(\mathbb{C})$ with the product

$$
\{x, y, z\}:=x \bar{y}^{\top} z+\delta\left(z \bar{y}^{\top} x-z \bar{x}^{\top} y\right),
$$

where $x, y, z \in \mathcal{M}_{m, n}(\mathbb{C})$, is a $(-1, \delta)$-FKTS. Indeed, straightforward calculations show that (2.4) and (2.5) hold so $\mathcal{M}_{n, n}(\mathbf{C})$ with the involution $x \mapsto \bar{x}^{\top}$ is a $\delta$-structurable algebra. 
REMARK 3.4. By [28], the following construction of Lie superalgebras is obtained by the standard embedding method. If $U(-1,-1):=\mathcal{M}_{2 n, m}(\Phi)$ with the product (3.15) then the corresponding standard embedding Lie superalgebra is $\mathfrak{o s p}(2 n \mid 2 m)=$ $D(n, m)$ (as defined by [12] and [9]), hence the standard embedding Lie superalgebra of the anti-structurable algebra $\mathcal{M}_{2 n, 2 n}(\Phi)$ is $\mathfrak{o s p}(2 n \mid 4 n)$. Similarly, if $U(-1,-1):=$ $\mathcal{M}_{2 n+1, m}(\Phi)$ with the product (3.15) then the corresponding standard embedding Lie superalgebra is $\mathfrak{o s p}(2 n+1 \mid 2 m)=B(n, m)$ (as defined by [12] and [9]), hence the standard embedding Lie superalgebra of the anti-structurable algebra $\mathcal{M}_{2 n+1,2 n+1}(\Phi)$ is $\mathfrak{o s p}(2 n+1 \mid 4 n+2)$. Furthermore, the construction of these Lie superalgebras and the correspondence with extended Dynkin diagrams is the subject of the next section. The structure theory of anti-structurable algebras their Peirce decomposition (in analogy with $[21,35]$ ) will form the subject of future work.

3.2. Anti-structurable algebras and extended Dynkin diagrams. In this section we will deal with a correspondence of anti-structurable algebras and extended Dynkin diagrams.

Let $U:=\mathcal{M}_{l, l}(\Phi)$ with the product (3.15) and $\delta=-1$, that is,

$$
\{x, y, z\}:=x y^{\top} z-z y^{\top} x+z x^{\top} y .
$$

Then from the previous section this triple system is an anti-structurable algebra and a simple unitary $(-1,-1)$-FKTS by means of a variation of Theorem $2.26(\kappa$ is simple and nondegenerate). Hence by the methods of standard embedding associated with $U$ we can obtain the standard embedding Lie superalgebra from the following proposition; the Lie (super)algebras notations and extended Dynkin diagrams are those of [9].

Proposition 3.5. Let $(U,\{\}), U=\mathcal{M}_{l, l}(\Phi)$, be a simple unitary $(-1,-1)$ FKTS defined by formula (3.16) and $L(U)=\bigoplus_{l=-2}^{2} L_{l}$ be the corresponding standard embedding Lie superalgebra. Then $L(U), L_{-2} \oplus L_{0} \oplus L_{2}, L_{0}$ and the corresponding extended Dynkin diagrams with $\otimes$ roots deleted are

$$
\left\{\begin{array}{l}
L(U)=B(n, l) \\
L_{-2} \oplus L_{0} \oplus L_{2}=C_{l} \oplus B_{n} \quad \text { for } l=2 n+1, \\
L_{0}=A_{l-1} \oplus B_{n} \oplus \Phi H
\end{array}\right.
$$

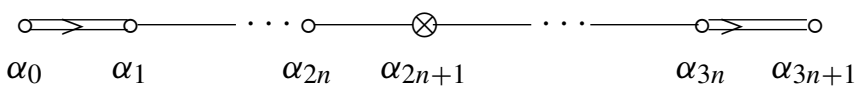

(ii) $\left\{\begin{array}{l}L(U)=D(n, l) \\ L_{-2} \oplus L_{0} \oplus L_{2}=C_{l} \oplus D_{n}, \quad \text { for } l=2 n . \\ L_{0}=A_{l-1} \oplus D_{n} \oplus \Phi H\end{array}\right.$ 


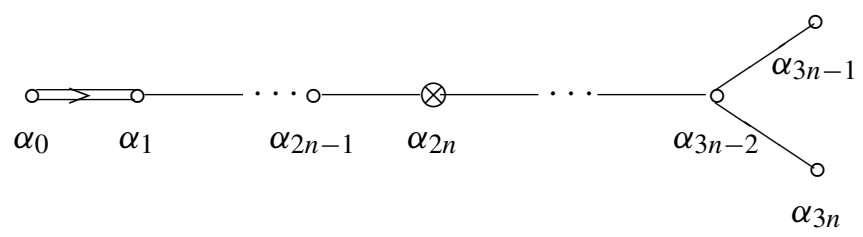

REMARK 3.6. These results mean that the correspondence between anti-structurable algebras and extended Dynkin diagrams is a useful concept for the structure theory of triple systems.

Finally, we state a conjecture.

Conjecture 3.7. Let $U:=\mathcal{M}_{p, q}(\Phi)$ and $L(U)=\bigoplus_{l=-2}^{2} L_{l}$ be the standard embedding Lie superalgebras of type (i) $B(m, n)$ or (ii) $D(m, n)$. Then there exist 5-tuples $(p, q, k, m, n) \in \mathbb{N}^{5}$ such that:

(i) $\quad p q=2(n+m-k) k+n$, where $1 \leq k \leq n+m$;

(ii) $p q=2(n+m-k) k+n-k$, where $1 \leq k \leq n+m-1$.

These extended Dynkin diagrams with $\otimes$ roots deleted are

(i)

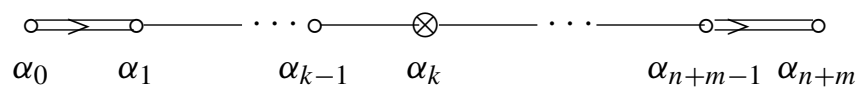

(ii)

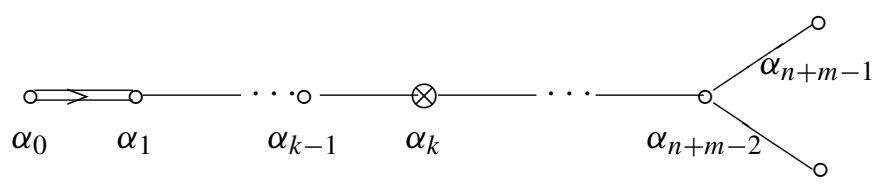

hence we have:

(i) $L_{-2} \oplus L_{0} \oplus L_{2}=C_{k} \oplus B_{n+m-k}$;

(ii) $L_{-2} \oplus L_{0} \oplus L_{2}=C_{k} \oplus D_{n+m-k}$.

\section{References}

[1] B. N. Allison, 'A class of nonassociative algebras with involution containing the class of Jordan algebras', Math. Ann. 237 (1978), 133-156.

[2] B. N. Allison, 'Models of isotropic simple Lie algebras', Comm. Algebra 7 (1979), 1835-1875.

[3] H. Asano, 'Classification of non-compact real simple generalized Jordan triple systems of the second kind', Hiroshima Math. J. 21 (1991), 463-489.

[4] H. Asano and K. Yamaguti, 'A construction of Lie algebras by generalized Jordan triple systems of second order', Nederl. Akad. Wetensch. Indag. Math. 42 (1980), 249-253.

[5] W. Bertram, 'Complex and quaternionic structures on symmetric spaces-correspondence with Freudenthal-Kantor triple systems', in: Theory of Lie Groups and Manifolds, Sophia Kokyuroku in Mathematics, 45 (eds. R. Miyaoka and H. Tamaru) (Sophia University, Tokyo, 2002), pp. 61-80.

[6] A. Elduque, N. Kamiya and S. Okubo, 'Simple $(-1,-1)$ balanced Freudenthal Kantor triple systems', Glasg. Math. J. 11 (2003), 353-372. 
[7] A. Elduque, N. Kamiya and S. Okubo, ' $(-1,-1)$ balanced Freudenthal Kantor triple systems and noncommutative Jordan algebras', J. Algebra 294 (2005), 19-40.

[8] J. R. Faulkner, 'Structurable triples, Lie triples, and symmetric spaces', Forum Math. 6 (1994), 637-650.

[9] L. Frappat, A. Sciarrino and P. Sorba, Dictionary on Lie Algebras and Superalgebras (Academic Press, San Diego, CA, 2000).

[10] N. Jacobson, 'Lie and Jordan triple systems', Amer. J. Math. 71 (1949), 149-170.

[11] N. Jacobson, Structure and Representations of Jordan Algebras, American Mathematical Society Colloquium Publications, XXXIX (American Mathematical Society, Providence, RI, 1968).

[12] V. G. Kac, 'Lie superalgebras', Adv. Math. 26 (1977), 8-96.

[13] N. Kamiya, 'A structure theory of Freudenthal-Kantor triple systems', J. Algebra 110 (1987), $108-123$.

[14] N. Kamiya, 'A construction of anti-Lie triple systems from a class of triple systems', Mem. Fac. Sci. Eng. Shimane Univ. 22 (1988), 51-62.

[15] N. Kamiya, 'A structure theory of Freudenthal-Kantor triple systems. II', Comment. Math. Univ. St. Pauli. 38 (1989), 41-60.

[16] N. Kamiya, 'On $(\epsilon, \delta)$-Freudenthal-Kantor triple systems', in: Nonassociative Algebras and Related Topics (Hiroshima, 1990) (World Scientific Publications, River Edge, NJ, 1991), pp. 65-75.

[17] N. Kamiya, The Construction of All Simple Lie Algebras over $\mathbb{C}$ from Balanced FreudenthalKantor Triple Systems, Contributions to General Algebra (Vienna, 1990), 7 (Hölder-PichlerTempsky, Vienna, 1991), pp. 205-213.

[18] N. Kamiya, 'On Freudenthal-Kantor triple systems and generalized structurable algebras', in: Non-associative Algebra and its Applications (Oviedo, 1993), Mathematiques \& Applications, 303 (Kluwer Academic Publications, Dordrecht, 1994), pp. 198-203.

[19] N. Kamiya, 'On the Peirce decompositions for Freudenthal-Kantor triple systems', Comm. Algebra 25 (1997), 1833-1844.

[20] N. Kamiya, 'On a realization of the exceptional simple graded Lie algebras of the second kind and Freudenthal-Kantor triple systems', Bull. Pol. Acad. Sci. Math. 46 (1998), 55-65.

[21] N. Kamiya, 'Examples of Peirce decomposition of generalized Jordan triple system of second order-Balanced cases', in: Noncommutative Geometry and Representation Theory in Mathematical Physics, Contemporary Mathematics, 391 (American Mathematical Society, Providence, RI, 2005), pp. 157-165.

[22] N. Kamiya and D. Mondoc, 'A new class of nonassociative algebras with involution', Proc. Japan Acad. Ser. A 84 (2008), 68-72.

[23] N. Kamiya and S. Okubo, 'On $\delta$-Lie supertriple systems associated with $(\epsilon, \delta)$-FreudenthalKantor supertriple systems', Proc. Edinb. Math. Soc. 43 (2000), 243-260.

[24] N. Kamiya and S. Okubo, 'A construction of Jordan superalgebras from Jordan-Lie triple systems', in: Nonassociative Algebra and its Applications, Lecture Notes in Pure and Applied Mathematics, 211 (eds. R. Costa and L. A. Peresi) (Marcel Dekker Inc., New York, 2002), pp. 171-176.

[25] N. Kamiya and S. Okubo, 'A construction of simple Jordan superalgebra of $F$ type from a JordanLie triple system', Ann. Mat. Pura Appl. (4) 181 (2002), 339-348.

[26] N. Kamiya and S. Okubo, 'Construction of Lie superalgebras $D(2,1 ; \alpha), G(3)$ and $F(4)$ from some triple systems', Proc. Edinb. Math. Soc. 46 (2003), 87-98.

[27] N. Kamiya and S. Okubo, 'On generalized Freudenthal-Kantor triple systems and Yang-Baxter equations', in: Proc. XXIV Inter. Colloq. Group Theoretical Methods in Physics, IPCS, 173 (2003) pp. 815-818.

[28] N. Kamiya and S. Okubo, 'A construction of simple Lie superalgebras of certain types from triple systems', Bull. Aust. Math. Soc. 69 (2004), 113-123.

[29] N. Kamiya and S. Okubo, 'Composition, quadratic, and some triple systems', in: Non-associative Algebra and its Applications, Lecture Notes Pure and Applied Mathematics, 246 (eds. L. Sabinin, L. Sbitneva and I. Shestakov) (Chapman \& Hall/CRC, Boca Raton, FL, 2006), pp. 205-231. 
[30] S. Kaneyuki and H. Asano, 'Graded Lie algebras and generalized Jordan triple systems', Nagoya Math. J. 112 (1988), 81-115.

[31] I. L. Kantor, 'Graded Lie algebras', Trudy Sem. Vect. Tens. Anal. 15 (1970), 227-266.

[32] I. L. Kantor, 'Some generalizations of Jordan algebras', Trudy Sem. Vect. Tens. Anal. 16 (1972), 407-499.

[33] I. L. Kantor, 'Models of exceptional Lie algebras', Soviet Math. Dokl. 14 (1973), 254-258.

[34] I. L. Kantor, 'A generalization of the Jordan approach to symmetric Riemannian spaces', in: The Monster and Lie Algebras (Columbus, OH, 1996), Ohio State University Mathematical Research Institute Publications, 7 (de Gruyter, Berlin, 1998), pp. 221-234.

[35] I. L. Kantor and N. Kamiya, 'A Peirce decomposition for generalized Jordan triple systems of second order', Comm. Algebra 31 (2003), 5875-5913.

[36] M. Koecher, 'Embedding of Jordan algebras into Lie algebras I. II', Amer. J. Math. 89 (1967), 787-816; 90 (1968), 476-510.

[37] W. G. Lister, 'A structure theory of Lie triple systems', Trans. Amer. Math. Soc. 72 (1952), 217-242.

[38] K. Meyberg, Lectures on Algebras and Triple Systems, Notes on a Course of Lectures Given During the Academic Year 1971-1972 (The University of Virginia, Charlottesville, VA, 1972).

[39] D. Mondoc, 'Models of compact simple Kantor triple systems defined on a class of structurable algebras of skew-dimension one', Comm. Algebra 34 (2006), 3801-3815.

[40] D. Mondoc, 'On compact realifications of exceptional simple Kantor triple systems', J. Gen. Lie Theory Appl. 1 (2007), 29-40.

[41] D. Mondoc, 'Compact realifications of exceptional simple Kantor triple systems defined on tensor products of composition algebras', J. Algebra 307 (2007), 917-929.

[42] D. Mondoc, 'Compact exceptional simple Kantor triple systems defined on tensor products of composition algebras', Comm. Algebra 35 (2007), 3699-3712.

[43] E. Neher, Jordan Triple Systems by the Grid Approach, Lecture Notes in Mathematics, 1280 (Springer, Berlin, 1987).

[44] S. Okubo, Introduction to Octonion and Other Non-associative Algebras in Physics, Montroll Memorial Lecture Series in Mathematical Physics, 2 (Cambridge University Press, Cambridge, 1995).

[45] S. Okubo, 'Symmetric triality relations and structurable algebras', Linear Algebra Appl. 396 (2005), 189-222.

[46] S. Okubo and N. Kamiya, 'Jordan-Lie superalgebra and Jordan-Lie triple system', J. Algebra 198 (1997), 388-411.

[47] S. Okubo and N. Kamiya, 'Quasi-classical Lie superalgebras and Lie supertriple systems', Comm. Algebra 30 (2002), 3825-3850.

[48] M. Scheunert, The Theory of Lie Superalgebras. An Introduction, Lecture Notes in Mathematics, 716 (Springer, Berlin, 1979), p. $\mathrm{x}+271$.

[49] J. Tits, 'Une classe d'algèbres de Lie en relation avec les algèbres de Jordan', Nederl. Acad. Wetensch. Proc. Ser. A 65 (1962), 530-535.

[50] K. Yamaguti and A. Ono, 'On representations of Freudenthal-Kantor triple systems $U(\epsilon, \delta)$ ', Bull. Fac. School Ed. Hiroshima Univ. 7 (1984), 43-51.

[51] E. Zelmanov, 'Primary Jordan triple systems', Sibirsk. Mat. Zh. 24 (1983), 23-37.

[52] K. A. Zhevlakov, A. M. Slinko, I. P. Shestakov and A. I. Shirshov, Rings that are Nearly Associative (Academic Press, New York, London, 1982).

\footnotetext{
NORIAKI KAMIYA, Center for Mathematical Sciences, University of Aizu, 965-8580 Aizuwakamatsu, Japan

e-mail: kamiya@u-aizu.ac.jp
} 
DANIEL MONDOC, Centre for Mathematical Sciences, Lund University, 22100 Lund, Sweden

e-mail: Daniel.Mondoc@math.lu.se

SUSUMU OKUBO, Department of Physics, University of Rochester, Rochester, NY 14627, USA

e-mail: okubo@pas.rochester.edu 